\title{
Thermodynamics of Aging and Heredity
}

\author{
Georgi P. Gladyshevev,2 \\ ${ }^{1}$ International Academy of Creative Endeavors, Moscow, Russia \\ ${ }^{2}$ N. N. Semenov Institute of Chemical Physics, Russian Academy of Sciences, Moscow, Russia \\ Email: academy@creatacad.org
}

Received 30 April 2015; accepted 21 May 2015; published 25 May 2015

Copyright (C) 2015 by author and Scientific Research Publishing Inc.

This work is licensed under the Creative Commons Attribution International License (CC BY). http://creativecommons.org/licenses/by/4.0/

c) (i) Open Access

\begin{abstract}
A brief review of the author's works in the sphere of thermodynamic theory of aging is presented. Particular attention is given to justification of the methods of classical and near-equilibrium "dynamic" thermodynamics used to assess the direction and extent of ontogenesis. It is noted that discovery of the law of temporal hierarchies and the substance stability principle made it possible to use quasi-equilibrium thermodynamics to describe aging of organisms and evolution of the living world. The review contains certain examples confirming thermodynamic direction of the origin of life and its development. The author states that supramolecular thermodynamics is the foundation of modern epigenetics. The review shows that the environment affects the composition and structure of the genetic apparatus as well as gene expression through the mechanisms of hierarchical thermodynamics. The author discusses the influence of "nutritive molecules" and other biological substances on tissue composition. It is noted that "nutritive molecules" can have an epigenetic influence on DNA and genetic apparatus as a whole. The author gives recommendations regarding nutrition and use of medicines from the perspective of the thermodynamic theory of aging. Though the author used simplifications and approximations when developing the thermodynamic theory of living beings' aging, the theory is in agreement with the detected correlation between changes in the specific Gibbs free energy of supramolecular structure formation and changes in the chemical composition of body tissues during aging. The author believes that hierarchical thermodynamics is the foundation of Darwinian natural selection.
\end{abstract}

\section{Keywords}

Aging, Thermodynamics, Genetics, Epigenetics, Nutrition

\section{Epigraphs}

"One of the principal objects of theoretical research in any department of knowledge is to find the point of 
view from which the subject appears in its greatest simplicity."

"The true and only goal of science is to reveal unity rather than mechanism."

J. Willard Gibbs

"The properties of living things are the outcome of their chemical and Poincare

"The properties of living things are the outcome of their chemical and physical composition and configuration."

Thomas Hunt Morgan

\section{Thermodynamic Theory of Aging}

All material systems and objects grow old. The phenomenon of aging observed in the inorganic world is usually taken for granted. Relatively easy construction and transformation of numerous objects in inorganic nature enabled scientists to discover a range of laws of nature. General laws of nature were formulated on the basis of the principles of simplicity and generality. Classical thermodynamics plays an important role in explaining the aging of objects in the inorganic world [1]. Many researchers followed the thermodynamic method trying to explain complex processes in the inorganic and living world "in an outburst of thought" [2]. However, this approach proved inapplicable to life phenomena as well as to other complex phenomena. Life sciences saw emergence of numerous aging theories, which take into account individual facts and regard them as aging causes. However, these facts are usually just signs or characteristics of aging. Such a situation resembles an attempt to find an elixir of youth or immortality. Of course, each of such hypotheses may contain some reasonable ideas, but they do not disclose generality-the essence of the phenomenon. One may notice that sometimes individual descriptive theories of a general nature insensibly take into account directional effect of thermodynamics on aging processes [3].

Thermodynamics of complex hierarchical systems is surely the driving force of aging [4]-[16]. However, the correct application of thermodynamics towards complex living natural systems is based on in-depth study not only of biology but also interdisciplinary sciences, especially physical chemistry [17]-[20], biophysical organic chemistry [21], and other physical disciplines [22] [23]. It is necessary to point out that many previous inept attempts of using thermodynamics to explain aging resulted in considerable misunderstandings, confusion and, finally, science discredit. It would be sufficient to point out erroneous ideas on "living dissipative structures" and numerous wordy irresponsible speculations on "entropy" or "negentropy" in natural systems [10] [11] [24][26].

It is much easier to understand the aging process from the viewpoint of thermodynamics if one studies this phenomenon from the perspectives of changes in chemical and supramolecular composition of body tissues [4]-[9]. At the end of the 70s of the last century, the author [4] applied methods of Gibbs' phenomenological thermodynamics towards near-equilibrium dynamic living systems and showed that body aging has a thermodynamic nature. It was found that aging is accompanied by changing of the averaged specific Gibbs function (Gibbs free energy) of formation of supramolecular structure of body tissues. The author claimed that this changing resulted from the second law of thermodynamics applicable to quasi-closed near-equilibrium supramolecular systems. The tendency to near-equilibrium intermolecular stable structure of all supramolecular formations and tissues of the body is accompanied by accumulation of chemically energy-intensive organic matter in the body. In the process of development (aging), the living body (as it has been known for a long time) loses water and acquires lipids (fat), proteins, polysaccharides and other energy-intensive chemical compounds. If we look at this phenomenon from the viewpoint of physical chemistry, it will be obvious that the system is enriched with organic matter that is obviously less stable than water, the amount of which significantly decreases with aging. It is worth mentioning that aging is accompanied by certain accumulation of stable inorganic substances, for instance, in bony tissues. However, this does not distort the general thermodynamic tendency of change in chemical composition of the body during aging.

In past years, the overwhelming majority of researchers believed that the specified body enrichment with relatively energy-intensive, poorly stable chemical substance contravenes thermodynamics. However, the author [4] claimed that it was wrong. According to him, the specified enrichment is a secondary effect. It results from the fact that energy-rich (relatively unstable) compounds have an increased affinity of supramolecular structure formation. Stability of the body's supramolecular structures increases as a result of the second law of thermodynamics. Accumulation of relatively unstable organic matters in the body tissues is accompanied by their gra- 
dual spontaneous decomposition, which is considerably accelerated under the influence of the environmental oxygen. All of this manifests itself in the body's metabolism cycle as well as in biological evolution on the whole.

In fact, the author claimed that the following held true for the supramolecular (intermolecular) and chemical (molecular) hierarchies in evolution and ontogenesis (during aging):

"The higher the supramolecular stability $\left(\Delta \Delta \tilde{\tilde{G}}^{\text {im }}<0\right)$ is, the lower the chemical stability $\left(\Delta \Delta \overline{\tilde{G}}^{\text {ch }}>0\right)$ is" or "The higher the chemical stability $\left(\Delta \Delta \overline{\tilde{G}}^{\text {ch }}<0\right)$ is, the lower the supramolecular stability $\left(\Delta \Delta \overline{\tilde{G}}^{\text {im }}>0\right)$ is". Here: $\Delta \overline{\tilde{G}}^{\text {im }}$-specific Gibbs free energy of formation of supramolecular (intermolecular) structure of body tissues, $\Delta \tilde{\bar{G}}^{\text {ch }}$-specific Gibbs free energy of formation of molecular (chemical) structure of body tissues. Symbol "- " means that value $\boldsymbol{G}$ is specific, and symbol “ " emphasizes the heterogeneous character of the system.

These rules correspond to the following schemes of works: article [4] (figure 1), book [7] (figure 4), and book [9] (figure 11).

The author named the presented regularity the "principle of chemical substance stability". Subsequently, the author extended this principle to all hierarchies of living matter and called it the "principle of substance stability”. Figure 1 shows the specified regularity.

The saw-tooth lines plotted against the curves emphasize that the fluctuation of the environmental parameters such as temperature, pressure, nutrition schedule, physical fields, change of day and night, change of seasons, etc., lead to variation of $\Delta \overline{\tilde{G}}^{\text {ch }}$ and $\Delta \overline{\tilde{G}}^{\text {im }}$. The organisms adapt to this variation only within the limits of the adaptive zone.

By now, the thermodynamic theory of aging (like the thermodynamic theory of life origin and biological evolution) has not undergone fundamental changes. Only some formulations were made more precise, explanations were added, certain misunderstandings were removed, and some terms and designations were added. As a rule, the author mentioned this in his works. These changes and explanations were meant to avoid confusion and possible misunderstandings.
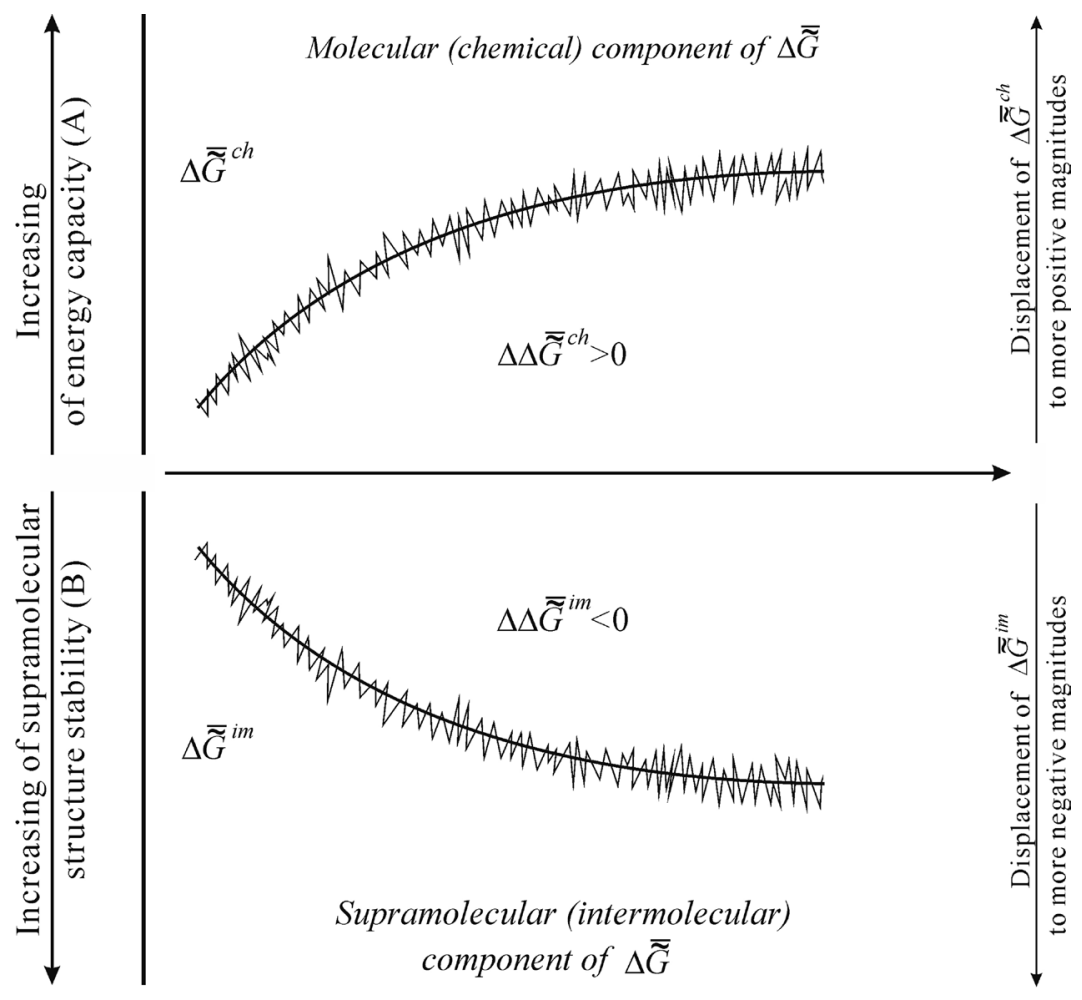

Figure 1. Scheme of changes in the specific chemical energy capacity of the biomass (biological tissue) (ch) - (A) and thermodynamic stability of its supramolecular structures during ontogenesis of living beings ( $\mathrm{im}$ ) - B. 
For specialists in physical chemistry, the described picture of aging is usually unsurprising. Aging of the body—non-stationary thermodynamic system—resembles aging of sorbent (adsorbent) of the equilibrium or rather quasi-equilibrium chromatographic column, which with time loses its activity due to accumulation of the "aging sorbent" that has a high affinity for the sorbing substance. Therefore it is clear why the author sometimes calls the thermodynamic mechanism of aging a "chromatographic" one, and the aging theory, the "chromatographic theory of aging” [27]-[29] (see: http://www.eoht.info/page/Aging).

Figure 2 diagrammatically shows change in specific Gibbs free energy of formation of supramolecular tissue structures in the process of body development and aging during ontogenesis.

Aging of a burning candlewick, rust formation in open flow-through systems and many other phenomena serve as common analogues of living beings' aging in the inanimate world [30].

Since biological evolution, phylogenesis and ontogenesis are thermodynamically directed, it is reasonable to consider these phenomena from the perspective of changes in specific values of formation of all hierarchical structures, using Gibbs equation — a generalized equation of the first and second laws of thermodynamics [5]-[7] [14]-[16] [30]. It is appropriate to mention that in our case, the generalized Gibbs equation takes into account spontaneous processes in the system and non-spontaneous processes stimulated in the system by the environment, which is a changing physical thermostat [5] of the complex quasi-closed system under study.

The well-known equation for differential of Gibbs function (free energy) may be represented in the following form [7] [14]-[17]:

$$
\mathrm{d} G^{*}=\sum_{i} \mathrm{~d} G_{i}^{*}=-\sum_{i} S_{i} \mathrm{~d} T_{i}+\sum_{i} V_{i} \mathrm{~d} p_{i}-\sum_{i} \sum_{k_{i}} x_{k_{i}} \mathrm{~d} X_{k_{i}}+\sum_{i} \sum_{k_{i}} \mu_{k_{i}} \mathrm{~d} m_{k_{i}}
$$

where: $G$ —Gibbs free energy; $T$-temperature; $S$ —entropy; $V$ —volume; $p$-pressure; $X$-any generalized force except pressure; $x$-any generalized coordinate except volume; $\mu$-chemical (evolutionary) potential; $m$ —mass of $k$-substance; work realized by the system is negative. Index $i$ pertains to the specific evolution, $k$ - to the component $i$ evolution. The superscript ${ }^{*}$ means that the behavior of a quasi-equilibrium complex system is considered.

The above equation is a generalized one since, (in principle) all interactions (inside and outside ones) of all structures on each hierarchical level are taken into consideration regardless of the scale of these interactions. It is logical to consider this equation as one with considerably divided parameters, symbolic or speculative, that can be efficiently used only in relation to everyone or adjacent hierarchies of structures.

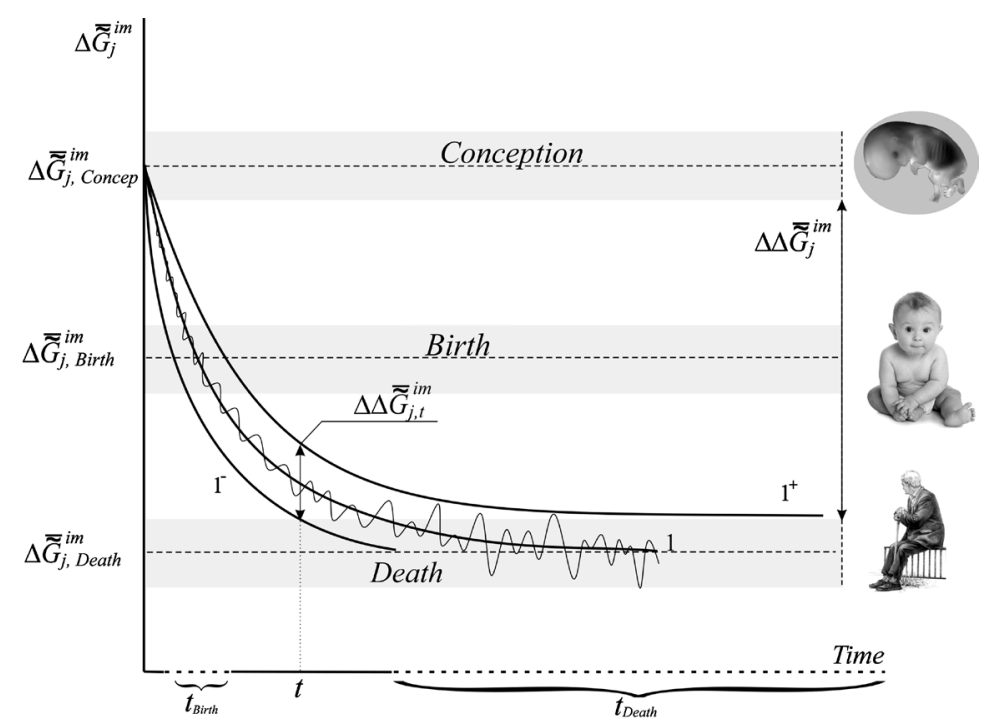

Figure 2. Diagram of variation in the specific Gibbs function (free energy) of formation of supramolecular tissue structures in the process of development and aging. Curve 1 indicates body development and aging under normal living conditions. Curves $1^{+}$and $1^{-}$refer to improved and bad living conditions correspondingly. The scheme conforms to the change in chemical composition of tissues. 
In this case, the Gibbs equation is considerably simplified in connection with negligibly small values of the majority of its isolated or individual members.

Apparently, the validity of the thermodynamics of life origin, evolution and living beings' aging was seriously confirmed due to understanding that hierarchical thermodynamics is the foundation of the evolution theory by Ch. Darwin and A. Wallace, which is based on the natural selection mechanism [13]. It is necessary to point out that this famous theory is defined more precisely now from the thermodynamic perspective and is extended to all hierarchical systems. Thus, hierarchical thermodynamics makes it possible to claim that it was an "aggregate of abiogenous molecules" or some "blocks of life", not a living being, what was the "common ancestor" of living bodies. It should also be mentioned that there were many works by chemists and biologists, which made it possible to realize that evolution and aging can be explained from the perspective of hierarchical thermodynamics. For instance, the author intuitively felt the thermodynamic direction of the living world development when he studied works by Aleksandr Oparin, Arthur Kornberg, Frederick Sanger, M. Ichas, Leslie Eleazer Orgel, M.S. Kanungo, Charles Tanford, C.R. Cantor, P.R. Schimmel, and other researchers [5]. It should be noted that recent outstanding works by Professor Kenji Sorimachi unambiguously confirmed the directionality of prebiological and biological evolution.

Thus, the thermodynamic theory of aging has started to use the idea of change in thermodynamic stability of molecular and supramolecular assemblies - structures of variable chemical and supramolecular composition. Such a correlation turned out to be useful, yet qualitative. The point is that thermodynamics cannot use absolute values of state functions (except for entropy) of elementary substances. Thermodynamics relies on the "standard reference level”, which is set as identical and equal to zero for all chemical elements (elementary substances) in the standard state. To determine precisely the change in stability of biological tissues when their chemical composition changes, it would be necessary to study the change in stability in accordance with stoichiometric equations of the processes. It is quite acceptable to compare the chemical stability and supramolecular stability of substances and their compositions of very similar atomic composition, for instance, oils, fats, and lipid fractions. Let us discuss this problem in more detail due to its importance.

One of the questions related to check of the thermodynamic theory of aging is as follows: "To what extent is it correct and reasonable to compare standard isobaric potentials of substance formation $\Delta \bar{G}_{f}^{0}$ (specific free Gibbs energies of formation) of different compounds with their relative thermodynamic stability?” This question, for instance, arises during study of the transition from chemical evolution to biological evolution.

Free energy (isobaric potential) of a chemical substance $\Delta \bar{G}_{f}^{0}$ is a free energy change for the reaction during which the substance in its standard state is formed from elements (elementary substances) taken in their standard states. Thus, the value of $\Delta \bar{G}_{f}^{0}$ may be regarded as stability of a substance in relation to the "reference point", which is represented by the "stability of original substances" taken in amounts corresponding to the stoichiometric reaction of the chemical substance formation. Therefore it is necessary to keep in view that the specified stability is a value related only to the "original" composition and quantity of elementary substances. Thus, it may be uninformative, incorrect or even senseless to compare the values of $\Delta \bar{G}_{f}^{0}$ pertaining to different chemical compounds from the viewpoint of their "absolute stability". However, we should point out that such comparison applied to several compounds that are "thermodynamically identical in composition" and used by the living nature during life origin and development shows that values of $\Delta \bar{G}_{f}^{0}$ for various "small groups of elements" differ considerably. This proves that the specified qualitative correlation is reasonable. Indeed, the values of the "standard stability" of compounds containing atomic groups "C, N", “ $\mathrm{H}, \mathrm{C}, \mathrm{N}$ ”, “C, O", "H, C, O", and "H, C, N, O" differ considerably and comply with our ideas on the relative physical and chemical tendency of chemical composition change in structures during aging and biological evolution.

Table 1 and Table 2 show values of standard isobaric potentials of formation (Gibbs free energies of formation), $\Delta \bar{G}_{f}^{0}$ of several nitrogen-containing and oxygen-containing substances under standard conditions [19]. One can see there are clear differences in the relative stability — between the free energy of formation of the specified nitrogen-containing substances (including initial substances-“life blocks"), $\Delta \bar{G}_{f}^{0}>0$, and the free energy of formation of the specified oxygen-containing substances (some metabolism products), $\Delta \bar{G}_{f}^{0}<0$. This proves, though intuitively, the reasonableness of conclusions on the tendency of chemical structure change in bodies during evolution and aging. All compounds represented in Table 1 are, under standard conditions, unstable with respect to the initial elementary substances they formed from. We can point out that the first three compounds mentioned in Table 1 (Cyanogen, Dicyanoacetylene, Hydrocyanic acid) are known to be relatively stable at high temperatures: their stability increases to a certain degree as the temperature rises. Relative stability 
Table 1. Standard potentials of formation at $25^{\circ} \mathrm{C}\left(\Delta \bar{G}_{f}^{0}\right)$ for some abiotic and other organic substances containing $\mathrm{C}, \mathrm{N}$ and $\mathrm{C}, \mathrm{H}, \mathrm{N}$.

\begin{tabular}{|c|c|}
\hline Substance & $\Delta \bar{G}_{f}^{0}$, ккал/моль \\
\hline $\mathbf{C}_{2} \mathbf{N}_{2}(\mathrm{G})$ Cyanogen & +71.03 \\
\hline $\mathbf{C}_{4} \mathbf{N}_{2}(G)$ Dicyanoacetylene & +122.1 \\
\hline HCN (G) Hydrocyanic acid & +28.7 \\
\hline $\mathbf{C H}_{2} \mathbf{N}_{2}(\mathrm{G})$ Diazomethane & +52.1 \\
\hline $\mathbf{C H}_{6} \mathbf{N}_{2}$ (G) Methylhydrazine & +42.5 \\
\hline $\mathbf{C}_{3} \mathbf{H}_{3} \mathbf{N}$ (G) Acrylonitrile & +46.7 \\
\hline $\mathbf{C}_{5} \mathbf{H}_{5} \mathbf{N}$ (G) Pyridine & +45.5 \\
\hline $\mathbf{C}_{6} \mathbf{H}_{7} \mathbf{N}(\mathrm{G})$ Aniline & +39.8 \\
\hline $\mathbf{C}_{5} \mathbf{H}_{5} \mathbf{N}_{5}$ (S) Adenine & +71.6 \\
\hline $\mathbf{C}_{4} \mathbf{H}_{4} \mathbf{N}_{2}$ (G) Pyrimidine & +67.2 \\
\hline
\end{tabular}

Table 2. Standard potentials of formation at $25^{\circ} \mathrm{C}\left(\Delta \bar{G}_{f}^{0}\right)$ for some metabolism products containing $\mathrm{C}, \mathrm{H}, \mathrm{O}$.

\begin{tabular}{|c|c|}
\hline Substance & $\Delta \bar{G}_{f}^{0}$, ккал/моль \\
\hline $\mathbf{C H}_{2} \mathbf{O}(\mathrm{G})$ Formaldehyde & -26.3 \\
\hline $\mathbf{C H}_{2} \mathbf{O}_{2}$ (L) Formic acid & -86.4 \\
\hline $\mathbf{C}_{2} \mathbf{H}_{2} \mathbf{O}_{4}$ (S) Oxalic acid & -167.6 \\
\hline $\mathbf{C}_{2} \mathbf{H}_{4} \mathbf{O}_{2}$ (L) Acetic acid & -93.1 \\
\hline $\mathbf{C}_{2} \mathbf{H}_{6} \mathbf{O}$ (L) Ethyl alcohol & -41.6 \\
\hline $\mathbf{C}_{3} \mathbf{H}_{8} \mathbf{O}_{3}(\mathrm{~L})$ Glycerol & -114.0 \\
\hline $\mathbf{C}_{4} \mathbf{H}_{10} \mathbf{O}$ (L) Butyl alcohol & -38.6 \\
\hline $\mathbf{C}_{6} \mathbf{H}_{12} \mathbf{O}_{6}$ (S) Glucose & -217.6 \\
\hline $\mathbf{C}_{12} \mathbf{H}_{22} \mathbf{O}_{11}$ (S) Sucrose & -369.1 \\
\hline
\end{tabular}

of the compounds listed in Table 2 is relatively high at low temperatures (their stability increases as the temperature drops). Compounds of the element groups “C, N”, “H, C, N" are mainly formed in non-spontaneous processes under the influence of relatively high energies on earth or in space. Compounds of the groups " $\mathrm{C}$, O” and "H, C, O" are mainly formed in spontaneous processes on earth or in space.

Apparently, informativeness of the specified correlation is connected with a certain feature uniformity of the atoms forming part of individual groups of elements used by living beings. These small groups are formed by three (or two) separate elements chosen from among the atoms of H, C, N, O as well as P, S- “basic elements” of living nature. The stated qualitative considerations are important and may serve as evidence for thermodynamic direction of evolution. However, there are general quantitative confirmations of thermodynamic direction of the living systems' evolution. On the whole, it is worth noting that the presence of nitrogen and sulfur atoms in "carbon-hydrogen-containing” organic molecules is a sign of relative instability of substances under normal conditions [19]. At the same time, the presence of oxygen in "carbon-hydrogen-containing” organic molecules usually shows relative stability of these chemical compounds [19]. Simultaneous presence of nitrogen and oxygen atoms in organic molecules should be considered a borderline case that determines the thermodynamic direction of metabolism processes. Apparently, it will be interesting to detect the physical basis of the specified 
tendencies more precisely in the future. It seems likely that the periodic law by Dmitri Mendeleev is a key to the riddle.

To detect aging directivity, it is primarily necessary to show how the supramolecular stability of molecular aggregates, including body's membranes, cells, tissues, changes during ontogenesis. The point is that this stability determines elasticity of supramolecular tissue structures, their metabolism rate, and other important features of body's biological structures.

As already noted, comparison of stability of substances with similar atomic composition, for instance, some lipids, oils, and fats, may be considered valid and quite precise. Based on this fact, let us proceed to justify comparative thermodynamic evaluation of aging and gerontological quality of food products.

Thus, as regards natural fatty acids, oils, and fats, we may accept to a good approximation that the initial atomic composition of these compounds (connected with the ratio of elements forming the substances) is almost identical. Therefore it is quite reasonable to use the free energy of formation of the specified substances $\Delta \bar{G}_{f}^{0}$ to compare their relative chemical stability under standard conditions. Conclusion on the supramolecular stability values for some oils and fats is confirmed by quantitative estimates made with the approximated GibbsHelmholtz-Gladyshev equation [31].

As applied to natural fats and oils, it can be written down as: [7]-[12]

$$
\Delta \overline{\tilde{G}}_{i}^{\text {im }}=\left(\Delta \overline{\tilde{H}}_{m_{i}}^{\text {im }} / T_{m_{i}}\right)\left(T_{m_{i}}-T_{\mathrm{o}}\right),
$$

where $\Delta \overline{\tilde{G}}_{i}^{\text {im }}$ is the Gibbs specific function or Gibbs specific free energy of the supramolecular or intermolecular formation of the condensed phase $i, \Delta \tilde{\tilde{H}}_{m_{i}}^{\text {im }}$ is the change of specific enthalpy during the solidification of natural fat (oil); $T_{m_{i}}$ is the pour (the congeal point) or melting point; and $T_{0}$ is the standard temperature (e.g., $25^{\circ} \mathrm{C}, 0^{\circ} \mathrm{C},-25^{\circ} \mathrm{C},-50^{\circ} \mathrm{C}$ ) at which values of $\Delta \tilde{G}_{i}^{\text {im }}$, and consequently $G P G_{i}$, are compared.

Estimations of change in free energy of reactions during abiogenesis, life origin and biological evolution $\left(\Delta \bar{G}_{r}^{0}\right)$ are known. They refer also to ontogenesis that, according to thermodynamics, repeats phylogenesis and evolution in general. Strict estimations unambiguously substantiate thermodynamic direction of bodies' aging, life origin processes and biological evolution. It would be sufficient to point out here that aging, just as chemical and biological evolution, is accompanied by "summary thermodynamic processes" which are characterized by free energy decrease [7]-[12] [28]. On the whole, it is worth mentioning that equilibrium and quasi-equilibrium thermodynamics has been widely used to detect direction of life origin, aging, and evolution [24] [32] [33].

Apoptosis is an issue of importance to thermodynamics. The question is whether this phenomenon exists. It is often said that aging is not genetically programmed. We believe that such understanding of aging process is incorrect from the viewpoint of hierarchical thermodynamics. Aging tendency, that is aging direction, is thermodynamically programmed. However, many apoptosis stages depend on changes in various environmental factors of all hierarchical body structures, including molecular and supramolecular environment.

Body aging is conditioned not only by direct conversion of its chemical and supramolecular compositions. Aging processes also depend both on chemical and physical environmental effects [30] [34] [35]. Hierarchical thermodynamics claims that all molecular and supramolecular body structures, including genetic apparatuschromatin, grow old in the process of ontogenesis. Higher hierarchy structures also grow old in the living world. In this case, DNA aging results in additional directional aging of genetic apparatus functions and body tissue aging. These processes produce a "snowball effect" bringing the body closer to death. Apparently, it would be reasonable to speak about genetically inherited, genetically modified, and adaptive tissue aging, which (tissue aging) does not depend on changes in DNA sequence. The first two types of aging can be connected with epigenetic mechanisms. Tissue aging can sometimes be regarded as an adaptive process that can be reversed. In any case, hierarchical thermodynamics admits there are various types and ways of aging of all hierarchical body structures.

Nowadays, some colleagues ask why the thermodynamic theory of aging has not yet become widespread and recognized. Primarily, this situation is likely to be connected with a stable fashion in science. As mentioned above, some researches still rely on the erroneous ideas on "living dissipative structures" by I. R. Prigogine and try to use "thermodynamics" of systems far from equilibrium. This "thermodynamics" uses the idea of "entropy" without an exact differential. Moreover, the specified "entropy" by Prigogine cannot be calculated or determined experimentally [26]. Some authors consider an issue of general entropy production by living beings. They believe this production leads to an increase in entropy of the whole planet. Of course, this value cannot be infor- 
mative in principle, and the corresponding hypothesis cannot be physically substantiated and checked. Besides, many science amateurs often discuss entropy and the second law of thermodynamics with an evident disregard of works by Rudolf Clausius, J. W. Gibbs, and other acknowledged scientists. At the same time, as mentioned above, it became obvious that in order to determine direction and degree of completion of ontogenesis (aging) and phylogenesis, one can use Gibbs free energy of formation or conversion of chemical and supramolecular body structures and make reasonable quantitative estimations.

Let us proceed to discussion of genetics and epigenetics of aging. According to thermodynamics, these spheres of knowledge are interconnected. Both genetic and epigenetic conversions influence aging and heredity. Hereditary apparatus represented by chromatin should be subject to aging like all body structures and tissues. However, the speed of its aging may considerably differ from that of tissue aging. The main reason for this is enhanced supramolecular stability of DNA. Hence, DNA structure should depend less on food nature in comparison with other body structures and tissues. The author proved this conclusion when discussing the speed of aging and update of various substances during metabolism. Thus, it was pointed out that the update rate of chemical composition of fat tissues exceeds the update rate of muscular tissues. This effect can be observed when the nature of the used diets changes [7]-[12].

The change of gene structure of the organism is connected with DNA sequence change, whereas epigenetic changes are mainly conditioned by conformational transformation of chemical and supramolecular structure of the entire genetic apparatus. Genetic apparatus transformation is generally connected with chemical and supramolecular processes accompanied by an indistinct free energy change of various scale. This makes it difficult to distinguish definitely between chemical and supramolecular transformations in genetic apparatus. Thus, chemical reactions-DNA methylation processes are believed to change epigenetic features of genetic apparatus. Changes in the chemical and supramolecular structure of chromatin are also classified as epigenetic processes. In general, terms and statements used in "epigenetics" sometimes have a different meaning. Probably, this is due to the fact that many researchers did not use the term "epigenetics” before. For instance, M. S. Kanungo and many of his colleagues avoided using this and similar terms in their research on aging and genetic apparatus transformation [36]. The author of the article did not also use this terminology when dealing with epigenetic problems from the perspective of supramolecular thermodynamics, in other words, epigenetic thermodynamics.

\section{Genetics and Epigenetics from the Perspective of Thermodynamics}

The overwhelming number of works on aging is dedicated to aging mechanisms. In other words, researchers try to answer the question "How we age?" However, it seems more important to know "Why we age?" The answer to the latter question should be given by thermodynamics, the driving force of everything that happens in the world. Unfortunately, many authors do not even try to answer this question. The very word "thermodynamics" is absent in the vast majority of publications. As already noted, this is connected with numerous misconceptions about the second law of thermodynamics and possibility of its use to understand the life phenomenon. Nevertheless, it is worth mentioning that thermodynamics is widely used in life sciences.

Aging of genes, chromatin, and tissues in ontogenesis goes in according with the laws of chemical and supramolecular thermodynamics of spontaneous processes and exterior environmental effects stimulating nonspontaneous body transformations. As already noted, these processes should be considered within the framework of a generalized Gibbs equation that is a generalized equation of the first and second laws of thermodynamics [24] [35]. In this case, the general Equation (1) is simplified because only the members related to molecular and supramolecular transformations should be taken into account.

DNA chemical structure is quite conservative in accordance with the principle of substance stability. According to thermodynamics, the type of DNA structure is predetermined by structure of abiogenous molecules which form and exist in space and on celestial bodies. It appears that the chemical structure of nucleobases, genes, and even genetic code should be the same (or single-type) over the entire universe. That is the conclusion we come to, at least intuitively, according to the indisputable laws of thermodynamics.

Thus, spontaneous aging of DNA under normal ontogenesis can apparently take place during unpairing of nucleic acid chains. In such cases, epigenetic (catalytic) transformation mechanisms should act. Another way of spontaneous DNA aging may be connected with the direct influence of chemical agents that quickly enter into a reaction with DNA chains or turn "epigenetic effect” into "chemical transformation" of structure of the very macromolecular sequence. The horizontal gene transfer should also be classified as a spontaneous process of 
DNA transformation. Non-spontaneous change in DNA sequence is influenced by various physical radiations and environmental forces. Influence of these factors can be treated as tropism phenomena [24] [35], which are represented in the form of individual members of a generalized Gibbs Equation (1). Numerous researches have been conducted in this sphere. However, it is not always easy to interpret them unambiguously. Nevertheless, we know for sure that the DNA structure changes during aging under the influence of various factors. Sometimes these factors are unpredictable. For instance, there are mutations caused by the paternal lifestyle and inherited by the children even if these mutations took place before their conception. Moreover, observations show that the germline mutations are present in all of the children's cells, including their own sex cells. This means that the paternal lifestyle "contains certain information" that influences the DNA of several generations, and not only of his direct descendants [37].

It is worth mentioning that the majority of gerontologists are convinced aging is connected with changes in the structure not only of DNA backbones, but also RNA and proteins. Apparently, Leonard Hayflick is right saying that the loss of precise or reliable information during aging results from accumulation of accidental exposures damaging essential molecules of DNA, RNA, and proteins.

Special attention should be paid to research by Kenji Sorimachi [38] who claims that evolution is based on the genome structure. He convincingly showed that Darwin's natural selection is doubtless an important factor in biological evolution and that all species originated from a single life source [39]. This conclusion is in agreement with the thermodynamic direction of evolution [4] [13] and equally refers to the aging process. Interesting ideas are voiced by DMR Sekhar [40] who endows the genome with certain brain functions. He believes that life is a state of matter with primary emergent properties such as self-programmability (genopsych), consciousness and free will, the origin of which is traceable to the genome. He thinks, what mind is to brain is as genopsych is to genome. In principle, these statements do not contradict hierarchical thermodynamics and aging theory. In any case, DMR Sekhar's ideas set us thinking about the ways in which thermodynamic information recorded in prebiological molecules and atoms is transferred to all hierarchical levels of the living matter.

It is important to point out the special role of epigenetic thermodynamics in aging processes. In this respect, we will take a closer look at the contemporary ideas on the direction of aging connected with epigenetics. Besides, for clarity sake, we will repeat some statements that have already been specified.

As noted above, it is often difficult to distinguish between genetic and epigenetic changes in the hereditary apparatus since these changes can accompany each other.

Generally, epigenetics is a branch of knowledge that studies inheritance of change in gene expression or cell phenotype, which is not connected with DNA sequence changes. Epigenetic changes may be preserved during division of somatic cells and transferred to the following generations. Epigenetic reactions include formation of various supramolecular structures with participation of DNA as well as changes in chemical composition of some nucleobases that do not change DNA sequence. The best studied processes are those of DNA methylation, which are not accompanied by change in the sequence of macromolecular chains of the very nucleic acid, but lead to changes in the supramolecular environment and its conformational structure. Of course, the DNA methylation reactions mainly connected with cytosine methylation proceed in accordance with the laws of chemical (molecular) thermodynamics. These processes are spontaneous and accompanied by a decrease in free energy.

Epigenetic mechanisms have been of interest to researchers for a long time. However, these mechanisms were studied from the perspective of structural and some chemical transformations that do not change the nucleotide sequence of nucleic acids. Only a few authors mentioned the thermodynamic direction of the processes. It became possible to study bodies from the viewpoint of supramolecular thermodynamics after researchers had realized the possibility of independent determination of the molecular and supramolecular temporal hierarchies and the principle of substance stability during evolution and aging of living beings had been formulated (1977).

It became obvious that supramolecular thermodynamics is the primary driving force of evolution and aging. Consequently, it turned out that conformations of biological polymers, proteins, saccharine, nucleic acids, and low molecular weight compounds could considerably change when their molecular environment changed. It meant that the body's genetic apparatus could be subject to transformation under the influence of small molecules penetrating the cells via the blood and skin. However, in previous years, epigenetic influence of low molecular weight substances on DNA or RNA conformation was not always called "epigenetic" since this usually referred to the possibility of using supramolecular (intermolecular) thermodynamics to any structures of the organism. As already noted, the author subsequently followed this tradition and did not use the term "epigenetics", though he continued his research in this sphere from the perspective of chemical and supramolecular thermody- 
namics.

In fact, supramolecular thermodynamics is the basis of epigenetics. For instance, in his monograph ([7] p. 67), the author wrote the following, "It makes sense to discuss from the thermodynamic perspective only the principal role played by DNA as the carrier of genetic information, whereas the properties and functions of DNA are also determined to a certain extend by the chemical and supramolecular structures framing the double spiral." Another monograph and a special section "On Supramolecular Thermodynamics of Genes and Aging" ([9], p. 99) as well as work [41] say, “... 'soft' anti-aging interference in DNA (RNA) supramolecular structures can be carried out by introducing chemically inert agents in nuclei and other cell elements. Such directed action does not promote changes in gene structure, but can affect processes of their adaptation to changes in the environmental conditions." Unfortunately, many researchers neglected achievements of the classical and hierarchical thermodynamics [5] [7] [21] and addressed the problem only from the perspective of empirical inductive methods.

In several works, the author frequently emphasized the advisability of using recommendations of hierarchical thermodynamics for gerontology of nutrition and treatment of various diseases, including cancer. Thus, work [12] said:

"Lastly, it is important to take into account, from the viewpoint of hierarchical thermodynamics, that anti-aging diets and many drugs can be used for prevention and treatment of cardiovascular diseases, cancer, and many other illnesses." We made this conclusion on the basis of thermodynamic estimations of the gerontological value of food products. Besides, we advanced a hypothesis about the role of dormant genes in the appearance of malignant neoplasm:

The principle of substance stability allows us to understand the influence of some chemical substances on the supramolecular structures of nucleic acids [6]. In the result of the action of such substances, ancient dormant genes (accumulated during the evolution of living beings) can awake. These genes can stimulate some types of cancer [28].

What are experimental proofs of the epigenetic thermodynamic direction of aging? The main proof of the thermodynamic nature of aging of a cell genetic apparatus is a directed rise in the chromatin melting temperature in various tissues of man and animals during ontogenesis. References to pioneer works in this sphere are given, for instance, in a monograph by M. Kanungo [36]. Subsequently, the author analyzed these and other similar results using the methods of hierarchical thermodynamics [5]. Analysis with the use of the Equation (2) showed that the rise in the chromatin melting temperature was unambiguously connected with an increase in the thermodynamic stability of chromatin supramolecular structures, which is accompanied by aging. There are proofs that changes in chromatin structure are connected not only with supramolecular transformations, but also with a change in the sequence and destruction of the main chains.

Lately, there have appeared articles which confirm predictions of the thermodynamic theory of life origin, evolution, and aging. It is worth paying attention to researches on the supramolecular structure of the genetic apparatus concerning epigenetic processes and gene expression. Thus, we can point out important works by Trivet O. Tollefsbol and his colleagues [42] [43]. Article [42] says that epigenetic processes are easily reversible. This circumstance is especially important from the viewpoint of treating epigenetically initiated diseases. The mentioned authors also showed that natural compounds could be epigenetically active in prevention and treatment of cancer. The presented experimental investigations [42] confirm the conclusions of the author of this article, which refer to thermodynamic supramolecular (epigenetic) mechanisms of aging. Besides, statements on the occurrence and treatment of cancer and some other diseases [12] [28] are becoming more grounded. Works by Professor V. K. Khavinson and his colleagues discuss the epigenetic effect of peptides, which he recommends as food supplements [44]. Conclusions of these works do not contravene supramolecular thermodynamics and are quite valid.

\section{On Epigenetics and Diets}

In works [10] [24] [29] [45]-[52], the author for the first time substantiated an idea that all components of the natural food consumed may be evaluated with the use of a gerontological value indicator based on thermodynamic parameters. The theory was based on the well-known facts of tissue enrichment with molecular fragments of the food used. For instance, systematic use of soft fats rather quickly increases the content of such (similar) fats in tissues of animals and humans and makes blood vessels elastic. At the same time, it was assumed that food constituents such as amino acids, sugars, fatty acids were directly used by the organism to synthesize pro- 
teins, polysaccharides, fats, and other "structural materials" of the body. Since the effects of change in tissue composition due to changes in food composition were considered reversible, they (effects) were not directly associated with DNA participation in adaptive transformation of the body's chemical structure. However, in the following studies, the author began paying attention to the fact that the food character influenced gene expression. He stated that this phenomenon was connected with the epigenetic action of the nutritive and similar molecules. Thus, we may assume that the character of food is reflected in the immediate adaptive changes in the body's composition and in inherited epigenetic changes. It is worth noting that adaptive and inherited changes explained by the principle of substance stability are interconnected, though they are manifested at different rates.

Products of digestion are components suitable for absorption and participation in metabolism. In the process of proteolysis, proteins break down into amino acids and, partially, small peptides. Fats break split into glycerin and fatty acids as well as monoglycerides and diglycerides. Carbohydrates split into monosaccharides, yet part of the breakdown products is represented by trisaccharides and disaccharides. "Young food" (biomass of young living beings) contains a relative surplus of fragments of complex molecules. Such food is also rich in physiologically active low-molecular substances, for instance, hormones and their simulators as well as vitamins, combined microelements, and other compounds that do not degrade in the digestive tract. In other words, the character of substances entering the blood stream greatly depends not only on the food type, but also on the age of organisms or plants used for food. It should be noted that a lack in the variety of physiologically active substances in food can be made up for by using various food supplements and medicinal preparations. As already mentioned, the author called substances entering the blood stream after food digestion "nutritive molecules". The nutritive molecules include unchanging fragments of various high-molecular food components as well as low-molecular substances that do not undergo considerable transformations during digestion. Nutritive molecules are, actually, unchanging molecules or parts of molecules that broke down during food digestion. In English, nutritive molecules may also be referred to as "nutritive particle molecules" [52].

Surely, high concentration of nutritive molecules of young organisms in the human blood promotes the synthesis of proteins, fats, carbohydrates, and other metabolites that correspond to somewhat revived tissues by their chemical composition, comparing with tissues of a patient eating biomass of ontogenetically old or evolutionarily well-developed ("phylogenetically old") organisms. In our opinion, the conclusion we make results from the hierarchical thermodynamic theory of biological evolution and aging of living beings.

The quantitative estimation of an increase in the stability of supramolecular structures during aging, for instance, by measuring melting temperatures of supramolecular formations (chromatin, tissue structures), is generally approximate. However, as already noted, the comparison of the stability of substances with slight differences in their general (atomic) composition, for instance, of many oils and fats, can be considered quite correct. Based on this fact, let us proceed to substantiate the comparative thermodynamic evaluation of aging and gerontological quality of food products.

Thus, in terms of some natural oils, fatty acids, and fats on their basis, we may assume to a good approximation that their original atomic composition (correlation of elements) is almost identical. Therefore, we may use the free energy of formation of the specified products $\Delta \bar{G}_{f}^{0}$ to compare their relative chemical stability. However, the correlation of the known data on $\Delta \bar{G}_{f}^{0}$ [53] shows this comparison may be ambiguous. In general, any comparison of the gerontological value of food products and their components should be made according to the standard procedures, and one should take into account possible "variety" in the samples under study. Apparently, it is most reasonable to compare the temperatures of melting or congelation of oils and fats for the practical purposes.

In conclusion of this section, we would like to pay attention to the fact that the thermodynamic theory of aging, including thermodynamic dietetics, is primarily based on physical chemistry, which relies (as a rule) on the models of ideal gases and ideal solutions. Physical chemistry also prefers studying transformations of individual chemical compounds. To transfer to research of complex systems requires various approximations. This applies particularly to the living systems which can be studied by examination of greatly simplified models. However, in spite of this, hierarchical thermodynamics makes it possible to detect regularities of aging processes, behavior of living systems and their evolution.

\section{Some Practical Recommendations}

From the perspective of the thermodynamic theory of aging, it is advisable to take into account gerontological 
value indicators of food products and medicinal preparations, gerontological purity of drinking water [10]-[12] [24] [45]-[48] [52]. However, in practice, it is convenient to follow the recommendations listed below.

To use as much as possible clean drinking water, the consumption of which is not contrary to medical indications.

To eat biomass of phylogenetically young (ancient) species of plants and animals—relatively young food (for instance, algae, some species of fish and amphibians).

To eat biomass of ontogenetically young plants and animals—relatively young food (germinated seeds, animals' germs, maggots, roe, young mammals, juvenile fish, chicks, etc.).

Preference should be given to:

Seafood, especially products of cold seas and rivers;

Biomass of plants and animals growing and living in cold regions: the extreme northern and southern areas of the planet, highlands;

Fats and oils with low melting points (algae oil, flax seed oil, cedar wood oil, sunflower oil, corn oil, soybean oil).

In case of propensity for diabetes and some other pathology, it is advisable to prefer vegetables to fruits. Besides, it is recommended to minimize consumption of carbohydrate-containing products (for instance, bread and floury products, rice, potatoes).

It is recommended to use food extracts and medicinal extracts of young medicinal plants growing in cold regions.

It is recommended to avoid eating overdone and processed food products with carcinogenic properties. Avoid taking medicines and food supplements with a low gerontological value.

The specified recommendations agree with the centuries-old human experience.

There are especially evident connections between GPG or $G^{\mathrm{G}}$ gerontological value indicator [45]-[48] and known experimental observations of human aging. $G^{G}$ indicator is estimated via the specific free energy of lipid fraction formation in biological tissues or via the congelation temperature of the lipid fraction.

It is easy to ensure that the presented recommendations are well-grounded if we compare experimentally obtained medical recommendations with conclusions of thermodynamic dietetics that takes into account physical and chemical characteristics of food products. For instance, let us compare some dietary fatty acids with fats widely used by people. Table 3 shows the content of saturated, mono-unsaturated, and poly-unsaturated fatty acids in dietary oils and fats. Dieticians will immediately notice that they, as a rule, recommend eating mainly oils and fats that contain unsaturated, especially poly-unsaturated, compounds.

\section{Table 3. Fatty acids in dietary fats (g/100g) http://en.wikipedia.org/wiki/Fatty_acid.}

\begin{tabular}{cccc}
\hline Name & Saturated & Mono-unsaturated & Poly-unsaturated \\
\hline Lard & Animal fats & & 9.6 \\
Duck fat & 40.8 & 43.8 & 12.9 \\
Butter & 33.2 & 49.3 & 2.6 \\
& 54.0 & 19.8 & $\mathbf{1 . 7}$ \\
Coconut oil & Vegetable fats & & $\mathbf{1 . 6}$ \\
Palm kernel oil & $\mathbf{8 5 . 2}$ & 6.6 & $\mathbf{8 . 3}$ \\
Palm oil & $\mathbf{8 1 . 5}$ & 11.4 & 48.1 \\
Cottonseed oil & $\mathbf{4 5 . 3}$ & 41.6 & $\mathbf{6 0 . 7}$ \\
Wheat germ oil & 25.5 & 21.3 & 56.5 \\
Soybean oil & 18.8 & 15.9 & 11.2 \\
Olive oil & 14.5 & 23.2 & 57.8 \\
Corn oil & 14.0 & 69.7 & $\mathbf{6 3 . 0}$ \\
Sunflower oil & 12.7 & 24.7 & $\mathbf{7 2 . 1}$ \\
Safflower oil & 11.9 & 20.2 & $\mathbf{7 5}$ \\
Hemp oil & 10.2 & 12.6 & 24.8 \\
Canola/rapeseed oil & 10 & 15 & 64.3 \\
\hline
\end{tabular}


Figure 3 shows dependence of the gerontological value indicator of individual dietary oils and fats, $\boldsymbol{G P G}_{\boldsymbol{i}}$, from their congelation temperature, $\boldsymbol{T}_{\text {Cong }}$. These data were obtained by calculations according to Gibbs-Helmholtz-Gladyshev Equation (2) [7]-[9] [31]. $\boldsymbol{G P G}_{\boldsymbol{i}}$ values and the corresponding $\boldsymbol{T}_{\text {Cong }}$ values are shown in the form of large circles. The calculation results are depicted in such a way as to attract the reader's attention to the dependence of the shown indicators and several known factors of environment. Thus, the congelation temperature of the indicated food products and, consequently, $\mathbf{G P G}_{\boldsymbol{i}}$ indicator change considerably with a change in the concentration of components of various fats and oils in the food products. These changes depend on the age of the organisms, environmental temperature for plants and animals as well as other environmental conditions. For instance, a reference is made to the phenomenon of Ali Gazayev, which is connected with a change in the congelation temperature of sea-buckthorn berries and other plant fruits growing at different heights in mountainous areas https://gladyshevevolution.wordpress.com/.

Comparison of the data presented in Table 3 and on Figure 3 shows that the known nutritional and medical recommendations correspond with physical chemical calculations made on the basis of thermodynamic theory. At the same time, the theory specifies and supplements medical indications that are often of a purely qualitative nature.

Thus, when giving thermodynamic estimations of gerontological quality of fats and oils, it is convenient to rely on their congeal temperature in practice. For instance, from the viewpoint of the thermodynamic theory, sunflower oil usually has a better anti-aging effect than olive oil. As already noted, coconut and palm oils contain high-melting fractions of fatty acids that is why they do not have an anti-aging effect. These data are quite logical in terms of metabolism. Sunflower and soybean oils promote vessel elasticity and relatively quickly participate in the body's metabolism. Coconut and palm oils make vessels more fragile and are assimilated relatively

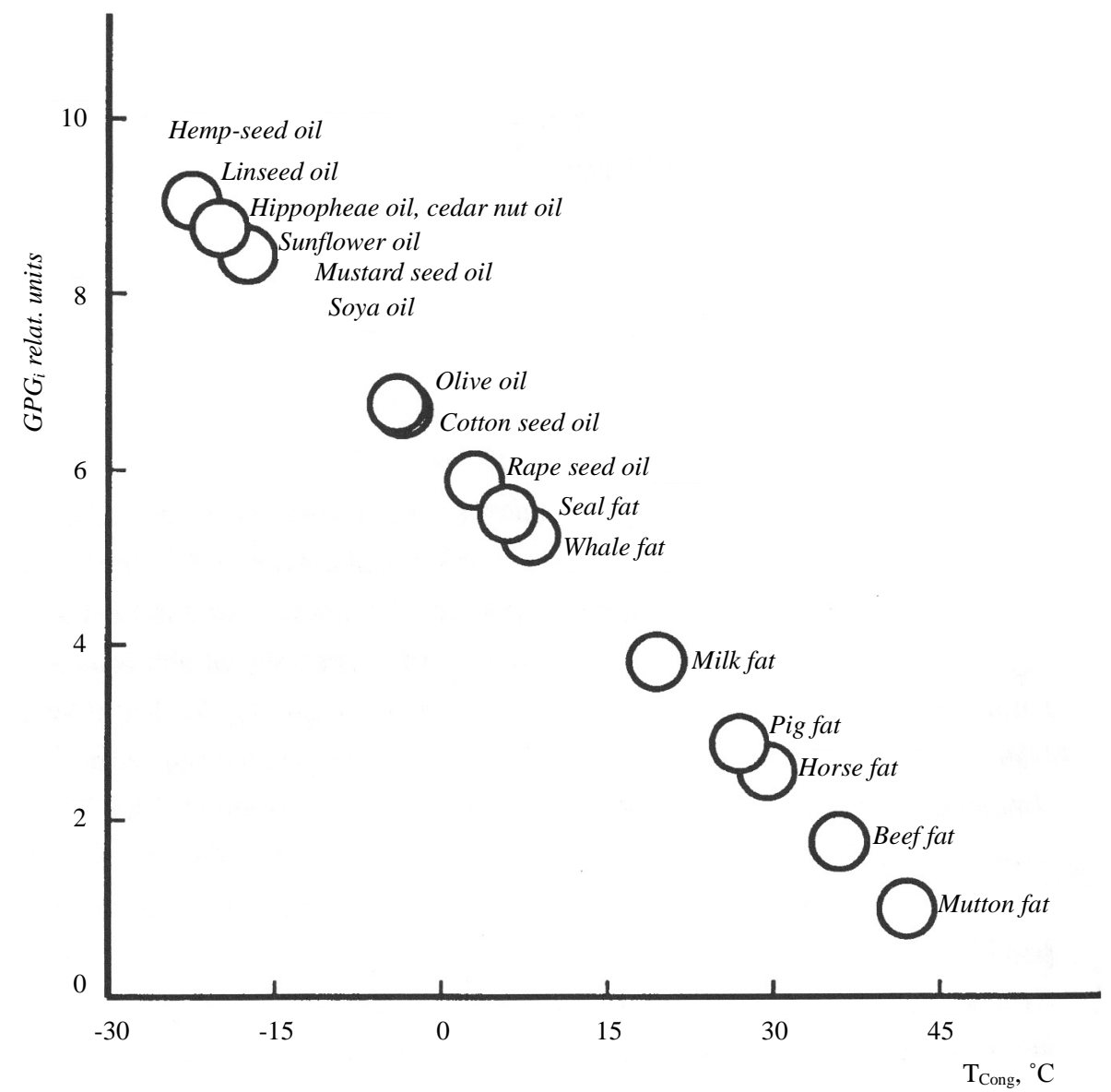

Figure 3. Dependence of the indicator of gerontological (anti-aging) value of oils and fats, $G P G_{i}$, on their congeal point, $T_{\text {Cong }}$. 
slowly. Therefore, coconut and palm oils are not wholesome in terms of anti-aging medicine. The same conclusions follow from Table 3: sunflower oil contains more poly-unsaturated fatty acids that olive oil. The corresponding conclusion can be made regarding coconut and palm oils. However, we would like to point out once again that anti-aging features depend on the temperature of plant growth and some other environmental characteristics. Anti-aging quality of oils and fats corresponds, according to patent [48], to the analogous quality of proteins and other ingredients of natural food products. Therefore, we may assume that $\mathbf{G P} \mathbf{G}_{\boldsymbol{i}}\left(\mathbf{G}^{\mathbf{G}}\right)$ indicator is a general anti-aging quality feature of a natural food product.

The recommendations presented in this section may be extended with account of all the factors considered by the thermodynamic theory of aging. We advise that people of all ages, especially senior citizens, use all of the given recommendations to the extent reasonable.

\section{Conclusions}

When studying life phenomena, including aging process, one can take into account the following general principle: Hierarchical thermodynamics brings together two ways of investigation of chemical and biological matter: the high road of classical thermodynamics by R. Clausius and J.W. Gibbs with the road of natural selection laid by Ch. Darwin and A. Wallace [13]. Despite the difficulty in making qualitative estimation of the thermodynamic direction of aging, in the author's opinion, it is worth noting that there are convincing proofs of the conclusions made by hierarchical thermodynamics [4]-[9] [54]-[61].

1) Thermodynamically favorable removal of water from developing living beings is accompanied by the accumulation of energy-intensive unstable organic substances in the tissues of organisms. This phenomenon leads to drying of body tissues, which is accompanied by its aging and brings to death.

2) Thermodynamically directed enrichment of body tissues with relatively stable supramolecular structures in ontogenesis during aging can be proved with known examples of adsorption (absorption) of energy-intensive substances (for instance, fats, oils, peptides, and proteins) due to their high affinity (adhesion) for organic adsorbents, that is supramolecular structures of body tissues. We may assume that the evaluation of change in the stability of the supramolecular structure of body tissues during aging (in spite of approximations made during calculations) is rather convincing. In fact, this evaluation (under standard conditions) is based on measurement of the average congelation temperature (melting temperature), for instance, of oils, fats or supramolecular structures of proteins, polysaccharides, and other similar structures of the living body.

These two experimentally confirmed statements form the basis for formulation of the substance stability principle. This principle predicts enrichment of an aging living being with an energy-intensive (relatively unstable) chemical substance as a consequence of the second law of thermodynamics-the specific Gibbs function of formation of the supramolecular structure of molecular associates, organelles, cells, and tissues of the body tends towards the maximum negative value.

There are grounds to believe that aging of the body under its normal development, from the perspective of genetic apparatus transformation, is connected with small, yet reliably identified changes in the molecular structure of genes. Epigenetic thermodynamic mechanisms exercise significant influence on aging; they condition changes in the chemical structure of nucleobases (without changes in DNA sequence), conformations of DNA and RNA as well as in the chemical and conformational structure of chromatin, various proteins and related compounds. In brief, nowadays we may claim that all epigenetic changes in the hereditary apparatus exert considerable influence on aging.

Thermodynamic approaches make it possible to detect the optimum living conditions, to choose diets, food supplements, and medicinal preparations for the directional change in expression of certain genes. This helps prevent and treat many diseases and prolong a healthy human life.

There are numerous works in the sphere of gerontology and geriatrics. The author wrote only about his own humble results concerning the thermodynamic theory of aging and provided some examples of studies consistent with this theory. Even though that nowadays the thermodynamic direction of aging is evident, there are a lot of tasks and questions which should be resolved.

\section{References}

[1] Gibbs, J.W. (1876) The Scientific Papers of J. Willard Gibbs: “On the Equilibrium of Heterogeneous Substances”, Vol. 1, Thermodynamics. Ox Bow Press, Connecticut. 
[2] Mendeleev, D.I. (1906) The Principles of Chemistry, Preface. Edition 8, S. - Petersburg. http://en.wikipedia.org/wiki/Dmitri_Mendeleev

[3] Anisimov, V.N. (2008) Molecular and Physiological Mechanisms of Aging. Nauka, St. Petersburg.

[4] Gladyshev, G.P. (1978) On the Thermodynamics of Biological Evolution. Journal of Theoretical Biology, 75, $425-441$. Preprint (the Priority), May, 1977. http://dx.doi.org/10.1016/0022-5193(78)90354-5

[5] Gladyshev, G.P. (1988) Thermodynamics and Macrokinetics of Natural Hierarchical Processes. Nauka Publishers, Moscow.

[6] Gladyshev, G.P. (1995) Thermodynamics of Hierarchical Systems. In: Zefirov, N.S., Ed., Chemical Encyclopedia, 4, Great Russian Encyclopedia, Moscow, 1062. http://www.xumuk.ru/encyklopedia/2/4371.html

[7] Gladyshev, G.P. (1997) Thermodynamics Theory of the Evolution of Living Beings. Nova Science Publishers, Inc., Commack, New York. http://creatacad.org/?id=35\&lng=eng

[8] Gladyshev, G.P. (1994) A Motive Force of Biological Evolution. Herald of the Russian Academy of Sciences, 64, 118-126.

[9] Gladyshev, G.P. (2003) Supramolecular Thermodynamics-The Key to the Realization of the Phenomena of Life (What Is Life in Terms of Physical-Chemist). 2nd Edition, Institute of Computer Science, Moscow - Izhevsk.

[10] Gladyshev, G.P. (2003) Thermodynamic Self-Organization as a Mechanism of Hierarchical Structures Formation of Biological Matter. Progress in Reaction Kinetics and Mechanism UK, USA 28, 157-188. http://dx.doi.org/10.3184/007967403103165495

[11] Gladyshev, G.P. (2004) Macrothermodynamics of Biological Evolution: Aging of Living Beings. International Journal of Modern Physics B, 18, 801-825. http://dx.doi.org/10.1142/S0217979204023970

[12] Gladyshev, G.P. (2007) Leonhard Euler's Methods and Ideas Live in the Thermodynamic Hierarchical Theory of Biological Evolution. International Journal of Applied Mathematics and Statistics (IJAMAS), 11, 52-68.

[13] Gladyshev, G.P. (2015) Natural Selection and Thermodynamics of Biological Evolution. Natural Science, 7, 117-126. http://dx.doi.org/10.4236/ns.2015.73013

[14] Sychev, V.V. (1973) Complex Thermodynamic Systems. Consultants Bureau, New York. http://dx.doi.org/10.1007/978-1-4684-1605-3

[15] Sychev, V.V. (1986) Thermodynamics of Complex Systems. Energoatomizdat, Moscow.

[16] Sychev, V.V. (2009) Complex Thermodynamic Systems. 5th Edition, Publishing House MEI, Moscow.

[17] Alberty, R.A. (1987) Physical Chemistry. 7th Edition, Wiley, New York.

[18] Silbey, R.J. and Alberty, R.A. (2004) Physical Chemistry. Wiley, New York.

[19] Stull, D.R., Westrum Jr., E.F. and Sinke, G.C. (1969) The Chemical Thermodynamics of Organic Compounds. John Wiley and Sons, Inc., New York. See Some New Data BioCyc, 2015.

[20] Denbigh, K.G. (1953) Thermodynamics of the Steady State. Methuen, London.

[21] Cantor, C.R. and Schimmel, P.R. Biophysical Chemistry. Vol. 1-3. San Francisco: W.H. Freeman and Co. (Pyc. Пep. М.: Мир, 1984-1985).

[22] Bogolubov, N.N. (1990) Selected Works. Part 1. Dynamical Theory. Gordon and Breach Science Publishers, New York.

[23] Sedov, L.I. (1980) The Thoughts on Science and on Scientists, Nauka, Russian Academy of Sciences, V.A. Steklov Mathematical Institute, Moscow.

[24] Gladyshev, G.P. (2014) The Thermodynamic Theory of Evolution and Ageing. Advances in Gerontology, 4, $109-118$. http://dx.doi.org/10.1134/S2079057014020064

[25] Gladyshev, G.P. (2014) Thermodynamics of the Origin of Life, Evolution and Aging. Advances in Gerontology, 27, 225-228. (In Russian)

[26] Lin, S.-K. (1999) Diversity and Entropy. Entropy, 1, 1-3. www.mdpi.org/entropy http://dx.doi.org/10.3390/e1010001

[27] Gladyshev, G.P. (2002) The Hierarchical Equilibrium Thermodynamics of Living Systems in Action. SEED Journal, 42-59.

[28] Gladyshev, G.P. (2006) The Principle of Substance Stability Is Applicable to All Levels of Organization of Living Matter. International Journal of Molecular Sciences, 7, 98-110. http://www.mdpi.org/ijms/papers/i7030098.pdf http://dx.doi.org/10.3390/i7030098

[29] Thims, L., Ed. (2014) EoHT. http://www.eoht.info/ 
[30] Gladyshev, G.P. (2014) The Thermodynamic Theory of Evolution and Ageing. Advances in Gerontology, 4, $109-118$. http://dx.doi.org/10.1134/S2079057014020064

[31] Kozlov, G.V. and Novikov, B.U. (2001) A Cluster Model for the Polymer Amorphous State. Physica-Uspekhi, 44, 681-724. http://dx.doi.org/10.1070/PU2001v044n07ABEH000832

[32] Alberty, R.A. (2011) Enzyme Kinetics, Includes CD-ROM: Rapid-Equilibrium Applications of Mathematica. Wiley, New York.

[33] Gladyshev, G.P. (2015) The Driving Force behind the Origin of Life. https:/gladyshevevolution.wordpress.com/article/thermodynamics-the-driving-force-behind-the-origin-of-life/

[34] Gladyshev, G.P. and Komarov, F.I. (1996) Hierarchical Thermodynamics and Gerontology. Herald of the Russian Academy of Medical Sciences, 6, 31-38.

[35] Gladyshev, G.P. (2013) Tropism as a General Phenomenon in the Universe. Can the Behavior of Systems of Different Nature to Describe “Single Formula?” International Symposium on Modern Problems of Higher Education and Research in the Field of Chemistry and Chemical Engineering, Kazakh National University, Almaty, 30-31 May 2013, 711.

[36] Kanungo, M.S. (1980) Biochemistry of Ageing. Academic Press, Waltham.

[37] Linschooten, J.O., Verhofstad, N., Gutzkow, K., Olsen, A.-K., Yauk, C., et al. (2013) Paternal Lifestyle as a Potential Source of Germline Mutations Transmitted to Offspring. The FASEB Journal, 27, 2873-2879. http://dx.doi.org/10.1096/fj.13-227694

[38] Sorimachi, K. (2009) Evolution from Primitive Life to Homo Sapiens Based on Visible Genome Structures: The Amino Acid World, Natural Science, 1, 107-109. http://dx.doi.org/10.4236/ns.2009.12013

[39] Sorimachi, K. (2010) Evolution Based on Genome Structure: The “Diagonal Genome Universe”. Natural Science, 2, 1104-1112. http://dx.doi.org/10.4236/ns.2010.210137

[40] Sekhar, D.M.R. (2014) Life, Consciousness and Evolution. https://dmrsekhar.wordpress.com/article/life-consciousness-and-evolution/

[41] Gladyshev, G.P. (1999) Supramolecular Thermodynamics Genome and Aging. Advances in Gerontology, 3, 30-31. (In Russian) http://www.medline.ru/public/art/tom4/art27.phtml

[42] Tollefsbol, T.O. (2014) Dietary Epigenetics in Cancer and Aging. Advances in Nutrition and Cancer Treatment and Research, 159, 257-267. http://link.springer.com/chapter/10.1007\%2F978-3-642-38007-5 15. http://dx.doi.org/10.1007/978-3-642-38007-5 15

[43] Daniel, M. and Tollefsbol, T.O. (2015) Epigenetic Linkage of Aging, Cancer and Nutrition. Journal of Experimental Biology, 218, 59-71. http://dx.doi.org/10.1242/jeb.107110

[44] Khavinson, V. (2014) Peptides, Genome, Aging. Advances in Gerontology, 4, 337-345. http://link.springer.com/journal/13329/4/4/page/1

[45] Gladyshev, G.P. (1999) Method for Measuring the Gerontological Value of Biologically Active Substances and Compositions, Mainly Food and Cosmetic Products. Canadian Patent No. 2,327,747.

[46] Gladyshev, G.P. (2004) Gerontology and Physical Chemical Nutrition. Advances in Gerontology, 13, 70-80. (In Russian)

[47] Gladyshev, G.P. (2008) Determination of Gerontological Value Natural Products. №2, 5. http://www.heraldrsias.ru/download/articles/00_Article_Gladyshev.pdf

[48] Gladyshev, G.P. (2009) A Method for Measuring the Value of Gerontological Foods on a Scale Gladyshev. (Russian Federation, Number 2,350,948) 27.03.2009. http://www.freepatent.ru/patents/2350948

[49] Gladyshev, G.P. (2007) The Thermodynamic Theory of Aging in Action: Medical Nutrition Recommendations for Patients of Any Age. In: Klats, R. and Goldman, R., Eds., Anti-Aging Therapeutics, Vol. IX, American Academy of AntiAging Medicine (A4M), Chicago, 135-152.

[50] Gladyshev, G.P. (2007) Hierarchical Thermodynamics—General Theory of Existence and Living World Development: Model of Aging and Anti-Aging Quality of Foods and Medicines. The 15th Annual World Congress on Anti-Aging Medicine \& Regenerative Biomedical Technologies, the Venetian Hotel, Las Vegas, 12-15 December 2007. http://www.prolibraries.com/a4m/?select=session\&sessionID=1152

[51] Gladyshev, G.P. (2010) Thermodynamics and Life. Herald of the International Academy of Sciences (Russian Section), 6-10. http://www.heraldrsias.ru/download/articles/01 Article Gladyshev.pdf

[52] Gladyshev, G.P. (2008) On the Mechanism of the Effect of Food on the Healthy Life. Advances in Gerontology, 21, 34-36. (In Russian) http://www.gerontology.ru/PDF_YG/AG_2008-21-01-1.pdf

[53] Larissa, P., Roberta Ceriani, C. and Guirardello, R. (2013) A Publication of the Italian Association of Chemical Engi- 
neering. Chemical Engineering Transactions, 32, 535-540. http://www.aidic.it/cet/13/32/090.pdf

[54] Gladyshev, G.P. (1999) On the Thermodynamics, Entropy and Evolution of Biological Systems: What Is Life from a Physical Chemist’s Viewpoint. Entropy, 1, 9-20. http://www.mdpi.com/1099-4300/1/2 http://dx.doi.org/10.3390/e1020009

[55] Mulligan, P.J., Koslover, E.F. and Spakowitz, A.J. (2015) Thermodynamic Model of Heterochromatin Formation through Epigenetic Regulation, Journal of Physics: Condensed Matter, 27, Article ID: 064109. http://iopscience.iop.org/0953-8984/27/6/064109/

[56] El-Diasty, F. (2011) Origin of Order: Emergence and Evolution of Biological Organization as a Problem in Thermal Physics. Advances in Life Sciences, 1, 30-39. http://article.sapub.org/10.5923.j.als.20110101.06.html

[57] Gladyshev, G.P. (2014) Life as a Phenomenon. International Journal of Applied Life Sciences and Engineering (IJALSE), 1, 97-98. http://www.ijalse.org/

[58] Gladyshev, G.P. (2014) Life as a Process of the Existence, Reproduction, and Aging of Polyhierarchical Systems. Advances in Gerontology, 4, 1-2. http://dx.doi.org/10.1134/S2079057014010032

[59] Gladyshev, G.P. (2014) Thermodynamics Optimizes the Physiology of Life. Philosophy \& Cosmology, 12, $152-162$. http://ispcjournal.org/journals/2014/PhC_2014.pdf

[60] Gladyshev, G.P. (2014) On the Change in the Isotope Compositions of Living Organisms during Aging and Evolution. Advances in Gerontology, 4, 107-108. http://dx.doi.org/10.1134/S2079057014020052

[61] Gladyshev, G.P. (2000-2015) Amplifications and Clarifications of the Theory. http://endeav.net/news.html http://gladyshevevolution.wordpress.com/

http://creatacad.org/?id=35\&lng=eng

http://creatacad.org/?id=48\&lng=eng 